\title{
La dimension plurielle du phénomène de radicalisation djihadiste violent
}

\section{André Dumoulin ${ }^{1}$}

À propos du radicalisme violent et des attentats multiformes associés, plusieurs explications ont dominé temporellement le champ des études. À chaque fois, un tropisme spécifique y fut associé en «ignorant totalement ou partiellement » les autres causes. Dans un premier temps, l'on se concentra sur l'explication géopolitique de la menace djihadiste pour ensuite alimenter le débat à propos des difficultés sociales ${ }^{2}$ explicitant le passage à l'acte mortifère. Il fut ensuite question de la dimension religieuse de l'action, y compris le pourquoi des actions suicides. L'explication fut ensuite élargie aux aspects psychologiques, psychanalytiques et psychiatriques («Sont-ils fous »?) pour, au final, en venir à la dimension culturelle et idéologique.

Dans cet espace fortement polémique s'affrontèrent plus particulièrement les adeptes de l'explication sociale et des discriminations explicitant sinon « excusant » les actions violentes (culture de l'excuse) associées à ces frustrations tandis que d'autres condamnaient cette vision «idéaliste » de «l'excuse » en invoquant plutôt une confrontation globale : « une guerre religieuse et civilisationnelle ». Et pour complexifier le tout, des études mirent en avant l'approche anthropologique. Mais le processus de radicalisation et d'islamisation ne peut être appréhendé par une seule discipline académique.

Ce n'est que plus récemment qu'un effort de synthèse pluridimensionnelle et pluriexplicatif est apparu, associant les différentes explications et surtout en considérant, sous la forme d'études empiriques et de manière spécifique le parcours de chaque radicalisé. «Les ressorts de ce phénomène et des trajectoires de radicalisation sont protéiformes, multifonctionnels et touchent des profils variés, aux origines sociales diverses, pas toujours pour les mêmes raisons $»^{3}$. Bien évidemment, derrière les spécificités personnelles nous

\footnotetext{
${ }^{1}$ Université de Liège et Institut royal supérieur de défense.

${ }^{2} \mathrm{Cf}$. à propos du sentiment de discrimination au début du processus de radicalisation, les modèles de Moghaddam (2005), de Wiktorowicz (2005) et Sageman (2008) où des «humiliations » locales et sentiment d'injustices personnelles sont alimentées aussi par des sentiments « d'outrage moral » causé par les conflits en zone musulmane (« guerre contre l'Islam »).

3 David THOMSON, Les revenants, Seuil, 2016, p. 87. Cf. aussi Xavier CRETTIEZ, Romain SÈZE, Bilel AININE, Thomas LINDEMANN, Saisir les mécanismes de la radicalisation violente : pour une analyse processuelle et biographique des engagements violents, Rapport de recherche pour la Mission de recherche Droit et Justice, Paris, avril 2017 ; Dounia BOUZAR, Etapes du processus de radicalisation et de déradicalisation, Practicies, projet de recherche, mai 2018.
} 
pouvons aussi tenter de poser le principe global de l'idéologie. Facteur qui semble aujourd'hui être la porte d'entrée à contrecarrer pour lutter contre ces actes barbares.

En dehors des aspects politiques, géopolitiques, religieux et idéologiques, nous retiendrons ici les pistes sociologiques et psychologiques.

\section{Aspects sociologiques}

D'une part, au sujet du recrutement de Daesh, il fut question de la mise en évidence de groupe de jeunes issus souvent d'un même quartier ${ }^{4}$, de groupes de copains refermés sur euxmêmes et très soudés ou de fratrie dont une grande partie est issue du petit commerce ou de $\mathrm{PME}^{5}$ avec certains liens avec la petite délinquance ${ }^{6}$. D'autant que pour Hirschi ${ }^{7}$, on commet le plus souvent des actes délictueux quand et parce que le lien social (attachement à des personnes de référence, engagement dans des centres d'intérêt, investissement dans l'action socialisée, croyances aux normes) qui unit l'individu à la société est affaibli ou rompu.

Il y a insistance sur une vision socio-économique défavorable de personnes laissées pour compte. La discrimination sociale, la discrimination à l'emploi et le refus d'accès au logement, aux lieux de loisirs associé au délit de faciès ou de consonance nominale poussent alors à une dévalorisation du sentiment d'appartenance nationale. Ceci explique l'usage de l'expression «jeunes d'origine musulmane », face à l'absence d'emploi des mots «jeunes d'origine catholique » pour parler de l'immigration italienne ou polonaise! Dès lors, nous pouvons constater l'utilisation par les imans radicaux du constat de la panne de l'ascenseur social et de stigmatisation médiatique pour miner le désir d'intégration et donner au djihad un effet de sirène et donner sens à l'engagement. Dès lors que le social est défaillant, le discours religieux peut devenir un produit de substitution. Le djihadisme devient alors un instrument de revanche social. Le jeune a alors « un compte à régler avec la société ».

Pour Olivier Roy, le djihadisme n'est « pas la preuve de la radicalisation de l'Islam, mais au contraire la manifestation de la violence naturelle des banlieues et des phénomènes urbains ('le djihadisme des loseurs'), maquillée d'Islam ${ }^{8}$ alors que Gilles Kepel, autre

\footnotetext{
${ }^{4}$ Luc VAN CAMPENHOUDT, « Jihadisme, le parcours des combattants », Sciences humaines, Paris, mars 2018, pp. 42 et sv. ; Gérard DAVET et Fabrice LHOMME, Inch'allah : l'islamisation à visage découvert, Fayard, 2018.

${ }^{5}$ Selon Scott Atran (2016).

${ }^{6}$ Christophe LAMFALUSSY, «Un djihadiste sur deux a un passé délinquant », dans La Libre Belgique, 14-16 août 2015.

${ }^{7}$ Travis HIRSCHIT, Causes of delinquency, University of California Press, Berkeley, 1969. Cf. Pierre THYS, Le rôle des groupes d'un point de vue criminologique, Module 52, CETR, ULg, 2017 (document non accessible).

${ }^{8}$ Le Monde, 24 novembre 2016 et 29 mars 2017. « Les jeunes radicalisés, s’ils s'appuient bien sur un imaginaire politique musulman (la Oumma des premiers temps), sont en rupture délibérée tant avec l'Islam de leurs parents qu'avec les cultures des sociétés musulmans ». (Olivier ROY, La peur de l'Islam, Textes rassemblés, éd. Le Monde l'aube, Paris, 2018, p. 13).
} 
spécialiste français de l'Islam contemporain, y voit derrière des causes sociales certes présentes, un enjeu davantage culturel issu d'une radicalisation religieuse: le salafisme qui offre un terrain de passage à l'acte terroriste ${ }^{9}$. En d'autres mots, «ils s'affrontent» sous la forme d'une question : y a-t-il radicalisation de l'Islam ou islamisation de la radicalité ? Reste la posture de François Burgat ${ }^{10}$ qui estime que le djihadisme est une des conséquences de la colonisation : les attentats sont des actes politiques reposant sur une lecture anti-impérialiste et tiers-mondiste. Benslama y voit lui la mise à disposition d'un surmoi attractif «un passe-partout de l'idéalisation à l'usage des désespérés d'eux-mêmes et de leur monde ${ }^{11}$.

Cependant, pour Marc Sageman, les candidats au djihad seraient plus fréquemment des personnes ayant un niveau d'éducation relativement élevé ${ }^{12}$. En vérité, les jeunes djihadistes n'appartiennent pas à une catégorie sociale particulière. Il s'agit de jeunes de quartiers défavorisés et marginalement de jeunes issus des classes moyennes ${ }^{13}$. Dans la majorité des cas, ils sont souvent déconnectés de leur famille (ou dans des processus relationnels faussés ou leurrés $)^{14}$ et «déconnectés » de leur pays. Il devient un exilé de l'intérieur et peut faire apparaître un moment une «radicalité apaisante » avec observation stricte de préceptes religieux et autres idéaux ou, à contrario, dans un processus de provocation et de recours verbal à la menace islamiste, jouant sur une conflictualité familiale et scolaire déstabilisatrice pour les parents et les enseignants ${ }^{15}$. Un passage par la case prison n'est pas exceptionnel où peut s'organiser des liens d'amitié et d'influence. La rupture nait ici d'une humiliation et « commettre un acte extrémiste, c'est un contrepoids indirect à une violence subie ${ }^{16}$, une 《 contre-humiliation ostentatoire infligée à la société ${ }^{17}$ avec la volonté de vivre parfois des moments de fraternité ensemble contre tous tout en gagnant de l'estime de soi. La complicité fraternelle apparaît comme un support au combat, une condition de résilience face à l'adversité, un moyen de cohésion interne, d'encadrement. Un peu comme dans l'analyse des

\footnotetext{
${ }^{9}$ « Pourquoi des jeunes d'ici deviennent kamikazes ? », entretien, Le Soir, 6 avril 2016.

${ }^{10}$ François BURGAT, Comprendre l'islam politique : une trajectoire de recherche sur l'altérité islamiste, 1973 2016, La Découverte, Paris, 2016.

11 Fethi BENSLAMA, Un furieux désir de sacrifice. Le surmusulman, Seuil, Paris, 2016, p. 13.

${ }^{12}$ Résilience ou comment combattre le terrorisme, dans Histoire \& stratégie, Areion, Paris, décembre 2014-février 2015 , p. 37.

13. Cf. aussi Michaël DANTINNE, Radicalisme et villes, CETR, ULg, 10 mars 2017 (document non accessible).

14 Dounia BOUZAR, «Le processus de radicalisation «jihadiste»: une triple dimension émotionnelle, relationnelle et idéologique », www.geostrategia.fr., 9 avril 2018.

15 Idem

${ }^{16}$ Ariane Bazan, ULB, Le Soir, 16 novembre 2015.

${ }^{17}$ Farhad Khosrokkhavar, Diploweb, 13 mai 2018.
} 
caractéristiques de la fraternité au combat des militaires ${ }^{18}$. Pour Michel Houellebecq, le terrorisme est un moyen de socialisation!

Dans une étude réalisée par Rik Coolsaet parue en 2016, les Belges qui sont partis rejoindre Daesh seraient majoritairement de jeunes délinquants ${ }^{19}$ répondant à un effet de mode, pour une quête de reconnaissance narcissique. Deux groupes seraient distinguables : le premier proche des milieux de la drogue, délits et violence urbaine (bandes) avec l'idée d'une intégration par le groupe radicalisé («gang Daesh ») comme exutoire à la violence ; le second, très minoritaire, moins repérable, se radicaliserait après le départ avec des motivations très personnelles d'un engagement qui l'est tout autant, pour donner un sens à la vie. La perspective de gains matériels (mariage, maisons, piscines, grosses voitures, pillage) ${ }^{20}$ stimulée par la propagande de Daesh peut probablement aussi attirer les deux groupes dans leur motivation au départ.

Aussi, le djihad se profile comme l'unique projet personnel et professionnel à leur portée, la voie traditionnelle - étude, emploi - ne fonctionnant pas positivement. Le déclassement en situation scolaire aboutit, par le djihadisme, à une forme de réhabilitation collective : la cause islamiste donne alors $\operatorname{sens}^{21}$ pour une minorité agissante. Pour d'aucun, il s'agit ici de la culture de l'excuse. L'accusation est alors portée vers ceux qui ont une lecture biaisée, auto-rassurante, faisant dire à certains qu'il y aurait une complicité entre une certaine gauche et les islamistes (Taguieff), nommée alors islamo-gauchiste ou fascisme vert ${ }^{22}$.

\section{Aspects psychologiques}

Bien des commentaires furent donnés par les citoyens considérant que les terroristes djihadistes étaient des fous isolés et relevaient de troubles psychiatriques. «Tout se passe comme si les sociétés occidentales ne pouvaient pas penser le djihadisme en dehors du registre de la psychiatrie » car ce serait contraire à «notre propre rationalité subjective $»^{23}$. On aimerait les savoir fous à lier et «se rassurer ». Certes, il est question parfois d'individus instables

\footnotetext{
${ }^{18}$ André THIEBLEMONT, «La culture de l'Armée de terre à l'épreuve de la modernité », Revue de Défense nationale, Paris, octobre 2010 ; Claude WEBER, "The soldier and his/her Society", Inflexions, "Cultures militaires, culture du militaire », La Documentation française, Paris, juin-septembre 2009, pp. 63 et sv.

${ }^{19} \mathrm{Cf}$. aussi Fabien TRUONG, Loyautés radicales. L'Islam et les « mauvais garçons » de la nation, La Découverte, Paris, 2018.

${ }^{20}$ Pierre THYS, La participation au combat ... avec ou sans retour, CETR, ULg, 2017 (document non accessible).

${ }^{21}$ Laurent BONELLI et Fabien CARRIE, Op.cit.

${ }^{22}$ André DUMOULIN, «La laïcité française et la loi sur le port de signes religieux dans les écoles publiques », La pensée et les hommes n 64 , numéro spécial « Francs-Parlers », Bruxelles, 2006.

${ }^{23}$ Laurent BARDIES, «Adversaires et ennemis. La diagonale des fous », Défense \& sécurité internationale, ${ }^{\circ}$ 133, Areion, Paris, janvier-février 2018, p 62.
} 
mentalement, de personnalités « paranoïaque et perverse ", schizophrènes ou faibles d'esprit ${ }^{24}$, immatures et psychopathiques ${ }^{25}$, mais la dimension comportementale ne relève pas majoritairement de la psychiatrie. Des problématiques psychiatriques sont néanmoins présentes chez un certain nombre d'embrigadés mais sans que nous soyons face à l'idée que les djihadistes sont tous $«$ fous $»^{26}$.

Pour David Thomson, les djihadistes ne sont ni des déséquilibrés, ni des damnés de la Terre, mais pour la plupart des jeunes désintégrés issus de l'immigration et habités par un projet théologico-politique. Les enquêtes indiqueraient davantage des fragilités narcissiques (faible estime de soi), une intolérance à la frustration sociale ainsi qu'une carence en affects ; y compris parfois des malaises intimes liés au genre. Par la violence, ils ont le sentiment d'exister et d'inverser les rôles : «d'insignifiants, ils deviennent des héros $»^{27}$. L'ère du vide est récupérée alors par l'islamisme radical en embuscade. En vérité, les profils d'engagement sont extrêmement hétérogènes tout comme les parcours individuels. Pour Serge Garcet, psychologue à l'ULg, «la construction du sentiment de privation relative peut être égoïste, c'est-à-dire individuelle (comparaison avec un autre individu), ou fraternelle et intégrer une dimension collective (mise en comparaison du groupe avec d'autres) ${ }^{28}$. La construction de cette frustration procède donc à la fois de sa propre incapacité à accéder aux ressources économiques et sociales, renforcement du sentiment d'injustice et édification de représentations stéréotypées. Au final, escalade possible vers l'acte violent par déshumanisation de l'autre envié.

Pour Serge Garcet «l'évolution se traduit dans l'espace public par une remise en question accrue du cadre législatif au nom de la référence religieuse perçue comme inaliénable. La revendication porte sur la nécessité d'obtenir de la part de la société des concessions par rapport au religieux selon un modèle de société où le politique et le religieux se confondent conformément au geste prophétique de Mohammed et caractéristique de l'islam politique. Par cette transformation, la blessure individuelle narcissique et identitaire se dissout dans la blessure symbolique et collective d'un idéal islamique blessé, générant ce que Benslama nomme les 'surmusulmans', qui revendiquent la restauration d'un islam souverain, éventuellement par le recours à la violence ».

\footnotetext{
${ }^{24}$ Nous nous rappellerons la réaction de l'avocat Sven Mary à propos de Salay Abdeslam : « Il a l'intelligence d'un cendrier vide».

${ }^{25}$ Marc LEPLONGEON, « Les fous d'Allah sont-ils malades », Le Point, 13 juin 2016.

${ }^{26} \mathrm{Cf}$. aussi l'analyse de Yann ANDRUETAN, « De la psychiatrisation du terrorisme », Inflexions, n ${ }^{\circ} 38$, Ministère des Armées, Paris, 2018, pp. 157 à 165. En France, il serait question de développer un réseau de «psychiatres coordonnateurs » dans les agences régionales de santé afin d'assurer un suivi précoce des personnes prises en charge. « Nous ne voulons ni psychiatriser la radicalisation ni criminaliser la maladie mentale, mais mieux éviter l'essor de profils mixtes » (Muriel Domenach, Secrétaire générale du comité interministériel de prévention de la délinquance et de la radicalisation, cité dans Le Monde, 10 avril 2019).

${ }^{27}$ Farhad KHOSROKHAVAR, entretien, «Ces jeunes qui se radicalisent », CNRS Le Journal, Paris, 12 mars 2015 .

${ }^{28}$ Serge GARCET, Entre posture victimaire et polarisation identitaire : la question de la privation relative au sein du processus de radicalisation, CETR, ULg, 2018 (non accessible).
} 
Par ailleurs, la posture des jeunes djihadistes doit être mis en relation avec les dimensions psychosociales et psychologiques de cette tranche d'âge constructiviste qu'est l'adolescence ${ }^{29}$, période de plus en plus large temporellement. C'est l'espace d'une sociologie « d'en bas », avec ses bricolages religieux adaptés à leur expériences et trajectoires concrètes.

Les deux tiers des « radicalisés » occidentaux ont entre 15 et 25 ans, période du passage de l'adolescence à l'âge adulte : moment charnière où ils veulent se mettre à l'épreuve pour découvrir ce qu'ils valent. Selon Serge Tisseron ${ }^{30}$, s'il y a eu difficulté à se mettre à la place d'autrui (transmission, éducation) et que cela n'a pas été réalisé entre 7 et 12 ans, nous pourrions voir apparaître rigidité, fragilité, appauvrissement affectif, absence d'émotion (athymhormie) et facilité à être endoctriné et devenir des «machines à tuer». Cela touche donc tous le tissu social, la fratrie, les mères, les pères absents (Farhad Khosrokhavar), et parfois des carences parentales. La radicalisation ne commencerait pas dans les mosquées ou dans les prisons mais dans le cadre familial là où l'autorité n'est pas présente. Sans autorité familiale, l'enfant va passer de l'insouciance mortifère au message délivré dans la rue (Yasmina Khadra).

Nous avons affaire souvent à des individus en quête d'une vie fantasmée, " glorieuse et héroïque » plutôt que fade et frustrante dans un environnement où l'individualisme est prégnant $^{31}$ et où nous savons aussi que selon une étude d'Unicef France parue en 2014, plus d'un jeune sur trois serait en souffrance psychologique. « Souffrance qui ouvre toutes grandes les portes aux recruteurs de Daesh $»^{32}$.

Jeunes qui sont nés après la fin du communisme et dans la mondialisation et ses questionnements, espace médiatisé où la violence est davantage affichée et où l'on perçoit un doute généralisé envers la parole publique renvoyant aux fakes news et autres théories du complot $^{33}$ auxquelles ils s'abreuvent sans esprit critique. Reste leur porosité extrême aux outils de propagande dont les contenus peuvent être tactiques et pragmatiques autant que stratégiques, politiques et théologiques ${ }^{34}$ ont utilisé plusieurs langues afin d'augmenter les recrutements étrangers, présenter l'EI d'abord puis en tant qu'État en construction avant de revenir à la lutte asymétrique et de guérilla.

\footnotetext{
${ }^{29}$ Fabien TRUONG, Loyautés radicales. L'islam et les « mauvais garçons de la nation », La Découverte, Paris, 2017.

${ }^{30}$ Serge TISSERON, conférence sur l'empathie, Université de Liège, 3 mai 2018.

${ }^{31} C f$. les travaux de Beck (2002), Gauchet (2013), Inglehart $(2005,2018)$ et de Vigarello (2014) sur le culte du moi, le plaisir immédiat, la recherche de reconnaissance.

${ }^{32}$ Rapport de Malek BOUTIH, « Génération radicale », Assemblée nationale, Paris, 2015.

${ }^{33} C f$. le dossier dans Diplomatie ${ }^{\circ} 73$, Areion, Paris, mars-avril 2015, pp. 37 et sv.

${ }^{34}$ Cf. Dounia MAHLOUly \& Charlie WINTER, A Tale of two Caliphates : Comparing the Islamic State's Internal and External Messaging Priorities, Report ICSR \& VOX Pol, King's College, London, 2016.
} 